\title{
Quantum anonymous voting with anonymity check
}

\author{
Dmitri Horoshkd* and Sergei Kilin \\ B.I.Stepanov Institute of Physics, NASB, Nezavisimosti Ave. 68, Minsk 220072 Belarus
}

(Dated: September 18, 2018)

\begin{abstract}
We propose a new protocol for quantum anonymous voting having serious advantages over the existing protocols: it protects both the voters from a curious tallyman and all the participants from a dishonest voter in unconditional way. The central idea of the protocol is that the ballots are given back to the voters after the voting process, which gives a possibility for two voters to check the anonymity of the vote counting process by preparing a special entangled state of two ballots. Any attempt of cheating from the side of the tallyman results in destroying the entanglement, which can be detected by the voters.
\end{abstract}

PACS numbers: 03.67.-a, 03.67.Dd

There is a general trend in the modern society to automatization and computerization of nearly all aspects of social life, including such subtle area as voting procedure in various contexts: from state governmental elections to decision making in rather small groups like parliaments or councils. As a consequence, a number of protocols for electronic voting have been developed and successfully applied in the last decades [1]. Since such protocols meet the information security problems of confidentiality, authentication and data integrity, they belong to the scope of the science of cryptography. In the modern electronic voting systems the information security is provided by means of public-key cryptography, guaranteeing secrecy under condition of limited computational resources of a potential adversary. With the advent of quantum computers [2] this condition becomes impractical, thus inspiring interest in unconditionally secure voting schemes and protocols. One perspective way to this end is connected to using quantum systems as information carriers, which proved to be successful for the development of unconditionally secure key distribution; the technology known as quantum key distribution [3] has reached presently the level of commercial realizations.

In the present work we propose a protocol of anonymous binary-valued voting involving $n$ persons (voters), each making a binary decision $b_{i} \in\{0,1\}$ and writing it on a ballot, and one person (tallyman) collecting the ballots and announcing the result $s=\sum_{i} b_{i}$. The proposed protocol possesses two security properties. The first property is the "anonymity of voting", meaning that the value of individual vote of the $i$ th voter, $b_{i}$, remains unknown to other voters, the tallyman, and any third party possibly monitoring the communication lines, unless $s=0, s=n, \min \{s, n-s\}$ voters cooperate, or the $i$ th voter discloses his decision. The second property may be called "non-exaggeration" and means inability of a voter to contribute a number different from 0 or 1 to the final sum $s$. The anonymity of voting protects the voters from a curious tallyman (and other parties), who may

*E-mail: dhoroshko@rambler.ru wish to learn who voted in which way, while the nonexaggeration protects the entire community from malicious voters who may wish to vote twice. The proposed protocol includes operations with quantum systems and provides unconditional security for both anonymity and non-exaggeration, which distinguishes it from other existing voting protocols, both quantum and classical, briefly reviewed below.

There is a possibility of guaranteeing the anonymity of voting unconditionally by means of conventional, i.e. classical cryptography, based on mathematical encryption. The corresponding voting protocol [4] is based on the principle of "sender untraceability", meaning such a communication scheme, where the recipient of several messages from several senders cannot determine which message came from which sender. Such a communication can be realized with unconditional security in the sense that the recipient is unable to establish any relation between the messages and the senders, even being in possession of infinite computational power [5]. However, the very property of untraceability creates, in the case of voting, an additional problem of determining which ballots come from legal voters, since illegal participants can send ballots in an untraceable way. This problem is solved by a special "ballot issuing" protocol (based on the technique of "blind signature") providing each legal voter with an "unforgeable" and "blind" digital ballot, which is used for sending a vote. The term "unforgeable" means that the ballot cannot be cloned, while the term "blind" means that the ballots are in no way related to the identities of legal voters. The ballots in the ballot issuing protocol are unconditionally "blind" but only conditionally "unforgeable", that is a person in possession of rich enough computational power is able to vote instead of legal voters. Thus, the property of "non-exaggeration" is realized by the overall voting protocol in a conditional way only.

A quantum protocol for anonymous surveying has been proposed recently, whose aim is to calculate the sum of individual contributions of the participants, like in the voting protocol, but with the contributions being real numbers from some limited interval rather than binary digits [6]. The protocol is based on bipartite entangled 
quantum state, whose relative phase carries the sum of contributions and can be measured only when two parts of the entangled system are gathered in the same location. Application of this protocol to binary-valued voting meets the problem of multiple voting by a dishonest voter, which is proposed to be solved by employing two non-cooperative ballot agents (tallymen). A similar protocol for quantum voting has been proposed [7], meeting the same problem of multiple voting, and various ways for solving it have been discussed requiring also an employment of two non-cooperative tallymen. Another similar quantum protocol based on multipartite entanglement and quantum Fourier transform has been proposed [8], which is also vulnerable to multiple voting and may be securely applied only under the assumption that the voter has no full control of the ballot at the time of writing the choice. All the mentioned quantum protocols provide unconditional anonymity of voting, but the property of "non-exaggeration" is reached on the cost of serious additional assumptions, which may be viewed impractical in some applications. In contrast, the present protocol provides in unconditional way both "eavesdropping detection", meaning non-zero probability of detection of any attempt to learn the distribution of votes among the voters, and "non-exaggeration", thus protecting the protocol from dishonest voters from one side and dishonest tallyman from the other side. Here we consider, like in Refs. [4, 6, 7, 8], a curious but not malicious tallyman, whose dishonest action is limited to learning the distribution of votes among the voters, but not to announcing a wrong value of the voting result $s$.

The protocol of voting is as follows. The participants are $n$ legal voters labelled by index $i=1,2, \ldots, n$ and a tallyman.

1. Each voter chooses either to vote or to check the anonymity of voting.

(a) In the case of voting the voter makes a binary decision $b_{i}$ with $b_{i}=0$ corresponding to "no" and $b_{i}=1$ corresponding to "yes" decision, and encodes it into a state of a two-level quantum system - qubit - playing the role of a ballot. Two orthogonal states $|0\rangle_{i}$ and $|1\rangle_{i}$ of a qubit (computational basis) are used for encoding of the corresponding value of $b_{i}$.

(b) In the case of anonymity check the $i$ th voter cooperates with the $j$ th voter, who also chooses to check the anonymity, and they together prepare their pair of qubits in the Bell state $\left|\Psi^{+}\right\rangle_{i j}$, where the Bell states are defined as

$$
\begin{aligned}
\left|\Phi^{ \pm}\right\rangle_{i j} & =\frac{1}{\sqrt{2}}\left\{|0\rangle_{i}|0\rangle_{j} \pm|1\rangle_{i}|1\rangle_{j}\right\}, \\
\left|\Psi^{ \pm}\right\rangle_{i j} & =\frac{1}{\sqrt{2}}\left\{|0\rangle_{i}|1\rangle_{j} \pm|1\rangle_{i}|0\rangle_{j}\right\} .
\end{aligned}
$$

2. After the encoding all voters send their qubits to the tallyman together with their identities. The latter excludes the possibility of voting for illegal participants and the possibility for legal voters to vote instead of their colleagues.

3. The tallyman collects all $n$ qubits and calculates the number of "yes" votes by applying to the $n$ qubit system the projector valued measure (PVM)

$$
\hat{P}(s)=\sum_{\pi}|m(s, \pi)\rangle\langle m(s, \pi)|,
$$

where $|m(s, \pi)\rangle$ is a product state of $n$ qubits in the computational basis, having exactly $s$ 1's in the order determined by the permutation variable $\pi$. The tallyman announces the voting result "s votes yes".

4. The tallyman sends the qubits back to the voters.

5. The voters make a ballot test.

(a) The voters, who have chosen to vote, measure their qubits in the computational basis. If the state of the qubit is different from the sent one, they state the ballot test failure.

(b) The voters, who have chosen to make an anonymity check, make a measurement of their pair of qubits in the Bell basis. If they get a result which is different from the Bell state $\left|\Psi^{+}\right\rangle_{i j}$, they state the ballot test failure.

A few comments to the protocol are necessary. In the present protocol the statement of the ballot test failure does not mean a public accusation of the tallyman, it is rather an information for the personal use by the voter (e.g. a council member).

The numbering with $\pi$ is as follows. All $n$-bit strings with exactly $s$ 's represent numbers $0 \leq m \leq\left(2^{n}-1\right)$ in binary notation. Let us sort the strings in increasing order of the corresponding numbers $m$ and label them with index $\pi$ taking consecutive integer values from 1 to $d_{s}=\left(\begin{array}{l}n \\ s\end{array}\right)$. In this way for any $0 \leq s \leq n$ we get a set of strings $m(s, \pi)$. The product state of $n$ qubits in computational basis with individual qubit states $\left|b_{i}\right\rangle_{i}, b_{i}$ being $i$ th bit from the string $m(s, \pi)$, is the state $|m(s, \pi)\rangle$. For example, in the case of 5 qubits $m(1,2)=00010$ and $|m(1,2)\rangle=|0\rangle_{5}|0\rangle_{4}|0\rangle_{3}|1\rangle_{2}|0\rangle_{1}$. The states $|m(s, \pi)\rangle$ are mutually orthogonal.

The projector given by Eq.(3) is a projector on the subspace of $n$-qubit system, having $s$ states $|1\rangle$ and $n-s$ states $|0\rangle$. Let us denote this $d_{s}$-dimensional subspace $V_{s}$. It is easy to see, that the subspaces corresponding to different values of $s$ are orthogonal and their sum is the entire state space of $n$ qubits. The states $|m(s, \pi)\rangle$ for given $s$ form a basis in $V_{s}$. The application of projective measurement Eq.(3) corresponds to measuring the number of "yes" votes, but not their distribution among the voters.

Let us see how the protocol guarantees the anonymity of voting. Consider an event $E(\mu)$ consisting in $2 k$ voters 
choosing to check the anonymity, $l$ voters voting "yes" and the rest voting "no". The state of $n$ ballot qubits collected by the tallyman is represented by a state

$$
|E(\mu)\rangle=\frac{1}{\sqrt{2^{k}}} \sum_{\pi \in \Omega(\mu)}|m(k+l, \pi)\rangle,
$$

where $\Omega(\mu)$ is a set of $2^{k}$ possible values of $\pi$. The state Eq. (44) belongs to the subspace $V_{k+l}$ and therefore is not affected by the projective measurement defined by Eq.(3). In the absence of errors the qubits sent back to the voters will always pass the ballot test in the Step 5 .

Now we consider a curious tallyman, who makes an additional measurement of qubits with the aim to obtain some information on who voted which way. The simplest way to learn the vote of the $i$ th voter is just to measure the $i$ th qubit in computational basis. If the $i$ th voter has chosen to vote, this attack passes unnoticed. But, if the $i$ th voter has chosen to check the anonymity with the $j$ th voter, their state $\left|\Psi^{+}\right\rangle_{i j}$ will be transformed into $|0\rangle_{i}|1\rangle_{j}$ or $|1\rangle_{i}|0\rangle_{j}$ with equal probabilities, and the subsequent Bell measurement will give results $\left|\Psi^{+}\right\rangle_{i j}$ or $\left|\Psi^{-}\right\rangle_{i j}$ with probabilities $\frac{1}{2}$. The latter result means the anonymity check failure. Thus, a curious tallyman faces a risk of being detected.

The possible attacks from a curious tallyman are in no way restricted to measurement of single qubits. The tallyman may wish to learn some partial information concerning the distribution of votes, for example, the total number of "yes" votes from a fraction of the voters. As it was mentioned above, we consider a curious but not malicious tallyman, who follows the protocol up to projecting the qubits onto a subspace $V_{s}$ and correctly determining the value of $s$. After that the tallyman may be interested in making an additional measurement of the qubits. To prove the unconditional "eavesdropping detection" we need to show that for any such measurement there is an event $E(\mu)$ for which the probability of ballot test failure is non-zero.

The most general type of measurement on a system of n qubits, which we call "the object", consists in attaching to them another quantum system of at least the same dimensionality (the measuring apparatus), making a unitary transformation $U_{O A}$ of both the object and the apparatus, and analyzing the resulting state of the apparatus [9]. Since the states $|m(s, \pi)\rangle$ for given $s$ form a basis in $V_{s}$, the unitary transformation can be determined by its action on the basis states:

$$
U_{O A}|m(s, \pi)\rangle_{O}\left|a_{0}\right\rangle_{A}=\sum_{\pi^{\prime}}\left|m\left(s, \pi^{\prime}\right)\right\rangle_{O}\left|a_{\pi \pi^{\prime}}\right\rangle_{A},
$$

where $\left|a_{0}\right\rangle_{A}$ is the initial state of the apparatus, and $\left|a_{\pi \pi^{\prime}}\right\rangle_{A}$ are its final states, generally not normalized. The subscripts $O$ and $A$ refer to the object and the apparatus respectively. Here we suggest that the measurement does not take the state of the qubits outside the subspace $V_{s}$, because otherwise the non-zero probability of ballot test failure is obvious. Thus, all possible measurements of the tallyman are parameterized by a set of states $\left\{\left|a_{\pi \pi^{\prime}}\right\rangle_{A}\right\}$.

To prove the property of "eavesdropping detection" of the proposed protocol, we need a result concerning the structure of strings $m(s, \pi)$ for given $s$. In the following we imply that $s$ is fixed and the positions of bits in a string are numbered from right to left.

Lemma. For given $s$ and any two numbers $1 \leq \pi, \pi^{\prime} \leq$ $d_{s}$, the string $m\left(s, \pi^{\prime}\right)$ can be obtained from the string $m(s, \pi)$ by a finite number of pairwise permutations of $0 s$ and $1 s$.

Proof. Let $w\left(s, \pi, \pi^{\prime}\right)$ be the set of positions of bits, which are different in $m(s, \pi)$ and $m\left(s, \pi^{\prime}\right)$. This set is a sum of two non-overlapping subsets: $w_{0}\left(s, \pi, \pi^{\prime}\right)$, containing the positions of bits which are equal to 0 in $m(s, \pi)$, and $w_{1}\left(s, \pi, \pi^{\prime}\right)$, containing the positions of bits which are equal to 1 in $m(s, \pi)$. The lengths of the subsets $w_{0}\left(s, \pi, \pi^{\prime}\right)$ and $w_{1}\left(s, \pi, \pi^{\prime}\right)$ coincide, because the number of $1 \mathrm{~s}$ in both strings is the same. Let us make a set of pairs $w_{01}\left(s, \pi, \pi^{\prime}\right)$ of the elements of both subsets, taking one position from $w_{0}\left(s, \pi, \pi^{\prime}\right)$ and one position from $w_{1}\left(s, \pi, \pi^{\prime}\right)$ in increasing order. The string $m(s, \pi)$ subjected to permutation of bits at positions defined by the set $w_{01}\left(s, \pi, \pi^{\prime}\right)$ gives the string $m\left(s, \pi^{\prime}\right)$.

Now we can proceed to proving the property of "eavesdropping detection" of the proposed protocol, which is based on the following theorem.

Theorem. For any measurement, defined by the apparatus states $\left\{\left|a_{\pi \pi^{\prime}}\right\rangle_{A}\right\}$, where is an event $E(\mu)$ for which the probability of ballot test failure is non-zero, unless all the states satisfy

$$
\left|a_{\pi \pi^{\prime}}\right\rangle_{A}=\left|a_{11}\right\rangle_{A} \delta_{\pi \pi^{\prime}},
$$

i.e. no measurement is done.

Proof. Let us suggest that the apparatus states contain a non-zero off-diagonal state $\left|a_{\pi \pi^{\prime}}\right\rangle_{A}, \pi \neq \pi^{\prime}$. Consider the event $E(\mu)$, where all voters have chosen to vote and the distribution of votes corresponds to the string $m(s, \pi)$. For this event the probability of ballot test failure is nonzero, because the qubits received by the voters are in a mixture having component $\left|m\left(s, \pi^{\prime}\right)\right\rangle$.

Now let us consider measurements with the apparatus states satisfying

$$
\left|a_{\pi \pi^{\prime}}\right\rangle_{A}=\left|a_{\pi \pi}\right\rangle_{A} \delta_{\pi \pi^{\prime}} .
$$

Consider any two values $\pi \neq \pi^{\prime}$. Due to the Lemma the strings $m\left(s, \pi^{\prime}\right)$ and $m(s, \pi)$ differ by finite number $k$ of pairwise permutations determined by the set of bit position pairs $w_{01}\left(s, \pi, \pi^{\prime}\right)$. Consider the event $E(\nu)$, where $k$ pairs of voters, determined by $w_{01}\left(s, \pi, \pi^{\prime}\right)$, have chosen to check the anonymity, and the rest have voted in a way described by the coinciding bits of $m\left(s, \pi^{\prime}\right)$ and $m(s, \pi)$. For this event the state of qubits before the measurement 
is a superposition of $2^{k}$ states of the type of Eq.(4), including $|m(s, \pi)\rangle$ and $\left|m\left(s, \pi^{\prime}\right)\right\rangle$. After the interaction with the apparatus these two components get factors $\left|a_{\pi \pi}\right\rangle$ and $\left|a_{\pi^{\prime} \pi^{\prime}}\right\rangle$ respectively, as indicated by Eq. (15), which leads to a non-zero probability of wrong result for Bell state measurement, unless $\left|a_{\pi \pi}\right\rangle=\left|a_{\pi^{\prime} \pi^{\prime}}\right\rangle$.

In summary, we have proposed a quantum protocol of voting, guaranteeing that each voter contributes only one vote and that any attempt of learning who voted which way is detectable with non-zero probability. The protocol is a cryptographic primitive, intended to be an element of a more complicated cryptographic system, providing complex security of voting, including, e.g. authentication of legal voters etc. The main weakness of the protocol is its inability to realize a guaranteed anonymity of a single voting act, providing only the probabilistic "eavesdrop- ping detection", which is useful for application to many voting acts during a rather long period. Another weakness is connected to the necessity of cooperation of voters having opposite decisions, i.e. most probably, belonging to different fractions. However, to our knowledge, it is the first voting protocol uniting the protection of the voters from a dishonest tallyman and the protection of the participants from a dishonest voter in unconditional way.

\section{Acknowledgments}

This work was supported by the project EQUIND performed within the $6^{\text {th }}$ Framework programme of European Commission.
[1] Secure Electronic Voting, ed. by D. Gritzalis (Kluwer, 2003).

[2] S. Ya. Kilin, in Progress in Optics, ed. E. Wolf, 42, 1 (2001).

[3] N. Gisin, G. Ribordy, W. Tittel, and H. Zbinden, Rev. Mod. Phys. 74, 145 (2002).

[4] D. Chaum, in Advances in Cryptology: EuroCrypt '88 Proceedings, edited by C. G. Günther, Lecture Notes in Computer Science (Springer-Verlag, Berlin), 434, 177-182
(1988).

[5] D. Chaum, J. Cryptology 1, 65 (1988).

[6] J. A. Vaccaro, J. Spring, and A. Chefles, Phys. Rev. A 75, $012333(2007)$.

[7] M. Hillery, M. Ziman, V. Buzek, M. Bielikova, Phys. Lett. A 349, 75 (2006).

[8] S. Dolev, I. Pitowski, B. Tamir, quant-ph/0602087 (2006).

[9] E. P. Wigner, Am. J. Phys. 31, 6 (1963). 